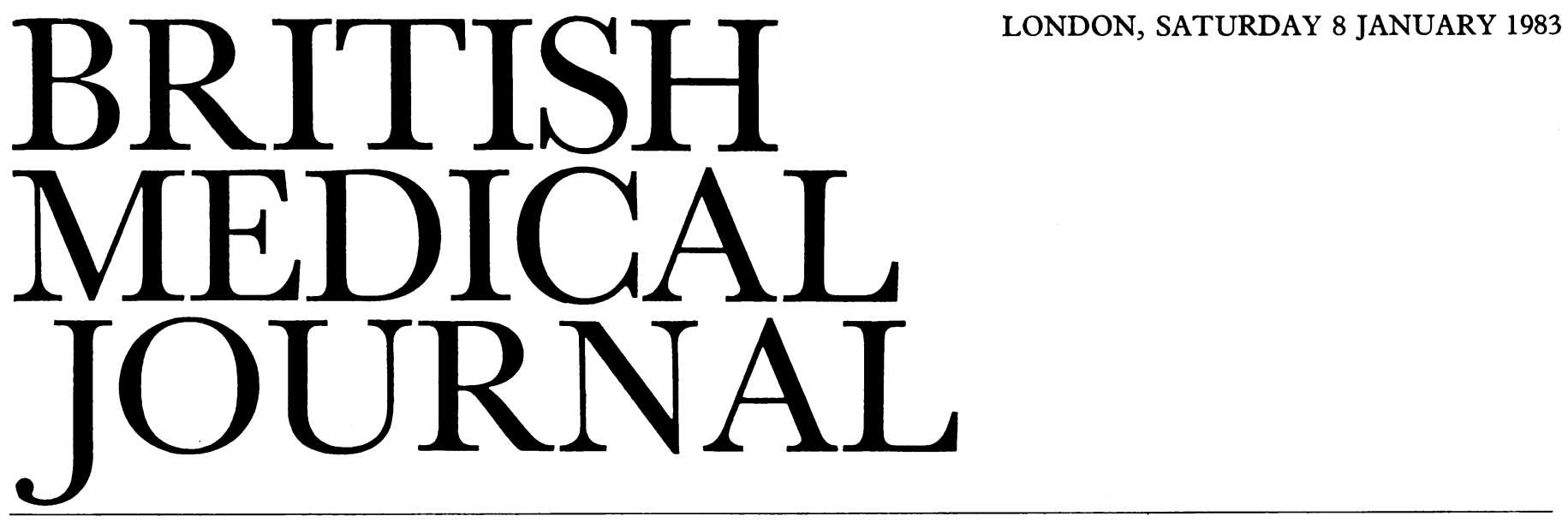

Oncogenes and the origins of human cancer

It is not unusual for scientists to make exaggerated claims for their discoveries, and it is even less unusual for journalists to exaggerate on their behalf. But there is some justification for the publicity that attended the recent publication in Nature ${ }^{12}$ of the discovery that human bladder cancer is associated with a point mutation in an identified gene. Nobody is claiming any immediate therapeutic or even diagnostic implications for this finding. What has been claimed is that it marks one of the high points in a remarkable year for fundamental research on the causes of cancer, and that at last we are seeing the vindication of all those tumour virologists who have over the past decade been insisting to increasingly deaf ears that the oncogenic viruses of animals must have something to tell us about carcinogenesis in man.

The scepticism of the past 10 years has no doubt been partly a reaction to the excesses of the early 1970s, when wild talk of vaccination against cancer accompanied claims to have identified human RNA tumour viruses-announced amid maximal furore only to vanish soundlessly soon afterwards, when they were unmasked as laboratory contaminants. Not that all human tumour viruses have come to such a bathetic end: the aetiological link between Epstein-Barr virus and Burkitt's lymphoma has held, and more recently a convincing human leukaemia virus has been extracted from the lymphocytes of patients with a rare and particularly nasty $T$ cell lymphoma. ${ }^{3}$ But on the whole people had stopped expecting viruses to have much bearing on human cancer, on the grounds that at best they might account only for a few rare tumours (chiefly lymphomas) whereas cancer in man is overwhelmingly a disease of the epithelium-the tissue most relentlessly exposed to environmental mutagens.

Identify the mutagens, the argument went, and we may be able to prevent cancer: more lives would be saved if everyone simply stopped smoking (for example) than if it were possible to vaccinate against every virus implicated in any human tumour. This general line of argument was advanced particularly cogently by John Cairns in the late 1970s when he was director of the Imperial Cancer Research Fund Mill Hill Laboratory; and it still holds. What is miraculous about the recent developments in molecular oncology-as Cairns himself points out in an editorial accompanying the papers on bladder cancer in Nature-is that they have made it possible to see carcinogenesis by mutagens and viruses in the same conceptual framework.
Interest in RNA tumour viruses first started to revive dramatically about two years ago, when the oncogenes responsible for the tumorigenic properties of the viruses were isolated, cloned, and shown to be homologous to genes in normal vertebrate cells. The viral oncogenes, of which about a dozen have been identified, fall into family groups now known to correspond to families of cellular genes that are highly conserved across all vertebrate species including man. Related oncogenes may, however, turn up in otherwise unrelated viruses; and, given that the life cycle of RNA viruses includes a phase of integration into the DNA of an infected cell, it has been assumed that the viruses must have picked up the cellular genes (known, deplorably, as proto-oncogenes) in transit. What they then do to make these normal and necessary genes oncogenic is so far only partly understood.

The products of most of the oncogenes (and their cellular equivalents) are unknown, but a few have been identified as protein kinases. The normal substrates of these enzymes have been very difficult to pin down, and research at the level of the DNA has proved much more rewarding. Here the main detectable difference between the viral and cellular genes is the rate at which they are transcribed.

Cellular proto-oncogenes are transcribed only at very low rates in normal cells, whereas viral oncogenes are transcribed at high rates under the influence of a powerful viral promotor. This observation led to the formulation of what is known as the dosage hypothesis of oncogenesis-which is more or less that you can have too much of a good thing. (Excessive concentrations of a protein kinase might, reasonably enough, be supposed to upset closely regulated cellular processes whose deregulation might lead to cancer.) A particularly ingenious experiment has provided some support for this idea: a cellular proto-oncogene was attached artificially to a viral promotor and cultured cells transfected with the hybrid DNA. Neither the viral nor the cellular sequence on its own had any effect, but the two together caused the cultured cells to become tumorigenic. $^{4}$

While these pieces of the cancer puzzle were being slotted together several other research groups were at work on a different corner of the picture. They began with the intention of isolating the oncogenes from human tumour cell lines-cell lines from tumours with no known or suspected viral aetiology. The approach was in principle rather crude: the DNA of the tumour cells was fragmented and used to transfect cultured 
mouse cells. Some of the mouse cells so transfected gave rise to tumours when injected into mice, and the fragments of DNA contained in such cells were deemed to contain an oncogene. On subsequent investigation the oncogenes they contained proved to be the cellular proto-oncogenes identified by tumour virologists. ${ }^{5}$

By this time the pieces of the puzzle were slotting together with almost audible clicks, and when Robert Weinberg and his colleagues at the Massachusetts Institute of Technology, Merck, and the United States National Institutes of Health, and Mariano Barbacid and his collaborators, also at the United States National Institutes of Health, came to sequence the mutant bladder cancer gene they described simultaneously in Nature $^{12}$ they were more than half expecting a direct confirmation of the dosage hypothesis. In other words, they were thinking in terms of a mutation outside the protein coding sequence of the gene and affecting a control region of the DNA so as to enhance transcription. In fact, however, they found a mutation in the protein coding sequence that substitutes a thymidine for a guanine, with the consequence that valine is incorporated into the amino acid chain of the protein concerned in place of a glycine.

They have made two points about this substitution, one of which bears on the dosage hypothesis. Firstly, the mutation of a guanine to a thymidine happens to be characteristic of the mutagenic action of some suspected bladder carcinogens. Secondly, glycine is the only amino acid that has no side chain. Its replacement by the bulkier valine could thus make a relatively large difference to the conformation of the proteinand hence is substrate specificity, or its affinity, or both. The result could be increased enzyme activity having much the same effect as increased dosage. Indeed, a re-examination of the sequences of the viral oncogenes in the light of the human gene sequence has shown that many of them have lost their glycine in favour of a residue with a side chain, at the same point in the chain.

More recently Mariano Barbacid and his collaborators have extended the work to a series of solid tumours, thus overcoming the objection that what they have found may be some bizarre artefact of tumour cell lines. Oncogenes have now been isolated from fresh tumours of lung, gut, and bladder, as well as a rhabdomyosarcoma. Interestingly, these oncogenes from tumours of different tissue origin all derive from the same proto-oncogene-the cellular homologue of the Kirsten murine sarcoma virus, or $\mathrm{Ki}$-ras.

Yet another line of parallel investigation has gained impetus from the discovery of proto-oncogenes. Many leukaemias are associated with chromosomal translocations, and in an article published in Nature last year George Klein, of the Karolinska Institute in Sweden, suggested that the effect of these translocations might be to transport a quiescent protooncogene into a region of high transcriptional activity. ${ }^{6}$ Specifically, in B lymphocytes transformed by Epstein-Barr virus, the translocations are known to concern chromosome 8 and one of the three chromosomes actively transcribing immunoglobulin chains. Klein guessed that chromosome 8 might contain a proto-oncogene whose translocation to the highly active immunoglobulin loci could account, under the dosage hypothesis, for the transformation of the cell. He has been triumphantly borne out: there is a proto-oncogene on chromosome 8 , and it is translocated to the immunoglobulin loci in transformed cells. ${ }^{7}$ This one, however, is a different oncogene: instead of being one of the ras family it belongs to a group whose viral homologue is the oncogene of the avian myelocytomatosis virus $(m y c)$. So it looks as though there may be some broad tissue specificity, at least distinguishing epithelial tumours from leukaemias. The details of tumour aetiology may thus vary - as one might expect; but clearly a wide range of phenomena are now covered by the same general conceptual umbrella.

So much for what fits. There is much that does not. For example, the transfection assay will identify only dominant oncogenes, whereas the transformed state is probably commonly recessive. Hence not surprisingly it has been possible to isolate oncogenes from only one sixth of all tumours investigated. A further weakness of the transfection assay is that so far it depends on a single line of cultured mouse cells-the NIH 3T3 line. NIH 3T3 cells are so precariously balanced this side of neoplastic transformation that the least disturbance is enough to tip them over. Thus the mouse $3 \mathrm{~T} 3$ cell is probably roughly equivalent to a human cell that has already undergone an unknown number of earlier steps on the road to carcinogenesis-now generally acknowledged to be a multistep process. The nature of the other steps may be as important as or more important than the mutation, translocation, or viral insertion that delivers the coup de grâce. In his editorial in Nature Cairns takes the view that the crucial question is which of these putative steps is most susceptible to environmental influence and thus in principle preventable.

In the more immediate future, however, the answers to some equally important questions are already accessible. For example, is the mutation detected in the bladder oncogene present only in tumour cells, or can it also be found in normal cells? And if it can, is it a predisposing factor for epithelial cancers? As Cairns remarks, the molecular biologist may soon be able to tell the epidemiologist what to look for.

Miranda Robertson

Staff Editor

Nature,

London WC2R 3LF

1 Tabin CJ, Bradley SM, Bargmann CI, et al. Mechanism of activation of a human oncogene. Nature $1982 ; 300: 143-9$.

2 Reddy EP, Reynolds RK, Santos E, Barbacid M. A point mutation is responsible for the acquisition of transforming properties by the T23 human bladder carcinoma oncogene. Nature 1982;300:149-52.

${ }^{3}$ Weiss R. A virus associated with human adult T-cell leukaemia. Nature $1981 ; 294: 212$.

1 Chang EH, Furth ME, Seolnick EM, Lowy DR. Tumorigenic transformation of mammalian cells induced by a normal human gene homologous to the oncogene of Harvey murine sarcoma virus. Nature 1982;297: 479-83.

5 Rigby PWJ. The oncogenic circle closes. Nature $1982 ; 297: 451-3$.

$6 \mathrm{Klein}$ G. The role of gene dosage and genetic transpositions in carcinogenesis: a review. Nature 1981;294:313-8.

${ }^{7}$ Marx JL. The case of the misplaced gene. Science $1982 ; 218: 983-5$.

\section{Maternal deaths in England and Wales 1976-8}

Intensive study of maternal deaths in England and Wales began in 1952. So efficient are the arrangements and so acceptable is the confidential nature of the inquiries that the ninth report (1976-8) has information on $99 \%$ of the deaths directly due to pregnancy or childbirth during the three years. " There were 227 "true" maternal deaths and a further 200 where death was associated with but not directly due to pregnancy and childbirth. An innovation in the report is the subdivision of these "associated" deaths into two groups: 97 were indirectly due to pregnancy in the sense that an 